\title{
A Rare Case of Vagococcus fluvialis Prosthetic Valve Endo- carditis and Aortic Root Abscess
}

\author{
Liam M Back ${ }^{1,2^{*}}$, Joseph Magdy ${ }^{1}$, Liam Guiney ${ }^{1}$, Roger Luo ${ }^{1}$, Akram Hussein ${ }^{1}$ and David Wong ${ }^{1}$ \\ ${ }^{1}$ The Sutherland Hospital, Caringbah, Australia \\ ${ }^{2}$ Prince of Wales Hospital, Randwick, Australia \\ *Corresponding author: Liam Marsden Back, The Sutherland Hospital, Kingsway and Kareena Road, \\ Caringbah NSW 2229; Eastern Heart Clinic, Prince of Wales Hospital, High Street, Randwick NSW 2031, \\ Australia, Tel: +612-9382-0700
}

\begin{abstract}
We describe a case of Vagococcus fluvialis infective endocarditis with associated aortic root abscess in a 50-year-old male on his second bioprosthetic aortic valve replacement. Only two cases of Vagococcus endocarditis have been recorded to our knowledge, and this case reinforces the fulminant nature of a rare organism.
\end{abstract}

\section{History of Presentation}

A 50-year-old male presented to our emergency department with 4 weeks of a traumatic back and neck pain with associated night sweats and anorexia. He had a history of intravenous (IV) drug use, but had abstained from injecting for the preceding 6 months, although had nasally ingested cocaine in the week prior to this presentation. Additionally, the patient had undergone tattooing in South East Asia two months prior to admission. At presentation, his blood pressure was recorded at $128 / 78$, heart rate 97 beats per minute, and temperature 37.6 degrees Celsius (99.7 degrees Fahrenheit). He displayed no peripheral stigmata of infective endocarditis and had no gross dental pathology. His cardiovascular examination was remarkable for dual heart sounds with a grade III ejection systolic murmur at the right upper sternal edge. General examination revealed point tenderness to palpation of both cervical and lumbar spine, with no focal neurologic deficit identified on detailed neurologic examination.

\section{Past Medical History}

Our patient has a history of several prior bacteraemic episodes in the context of IV drug use with significant cardiac comorbidity as a result. In 2016, our patient was diagnosed with Enterococcus faecalis infective endocarditis and underwent surgical aortic valve replacement (SAVR) with a bioprosthetic aortic valve $(25 \mathrm{~mm}$ Mosaic porcine valve). In late 2017 , he was diagnosed with Streptococcus parasanguinis and suspected infective endocarditis, and was managed conservatively with a prolonged course of IV antibiotics. In late 2018, he was diagnosed with prosthetic valve endocarditis secondary to Streptococcus vestibularis and underwent a second sternotomy and SAVR ( $25 \mathrm{~mm}$ Mosaic porcine valve). These prior episodes occurred in the context of ongoing active IV drug use.

Other relevant history includes Hepatitis C (Genotype 1a) diagnosed in 2016 in the context of injecting drug use which was yet to receive directed therapy.

\section{Differential Diagnosis}

The differential diagnosis included infective endocarditis, discitis and vertebral osteomyelitis.

\section{Investigations}

White cell count from admission was $13 \times 10^{\wedge} 9 / \mathrm{L}$ with neutrophil predominance, C-reactive protein 43 $\mathrm{mg} / \mathrm{L}$ and erythrocyte sedimentation rate $85 \mathrm{~mm} / \mathrm{h}$. Haemoglobin measured at $111 \mathrm{~g} / \mathrm{L}$ and platelets $144 \times$ $10^{\wedge} 9 / \mathrm{L}$. Electrocardiograph revealed sinus rhythm with $1^{\text {st }}$ degree AV block (PR interval $330 \mathrm{~ms}$ ) (Figure 1). CT whole spine demonstrated no epidural collection, no

Citation: Back LM, Magdy J, Guiney L, Luo R, Hussein A, et al. (2021) A Rare Case of Vagococcus fluvialis Prosthetic Valve Endocarditis and Aortic Root Abscess. Int J Clin Cardiol 8:213. doi.org/10.23937/23782951/1410213

Accepted: January 14, 2021: Published: January 16, 2021

Copyright: (C) 2021 Back LM, et al. This is an open-access article distributed under the terms of the Creative Commons Attribution License, which permits unrestricted use, distribution, and reproduction in any medium, provided the original author and source are credited. 


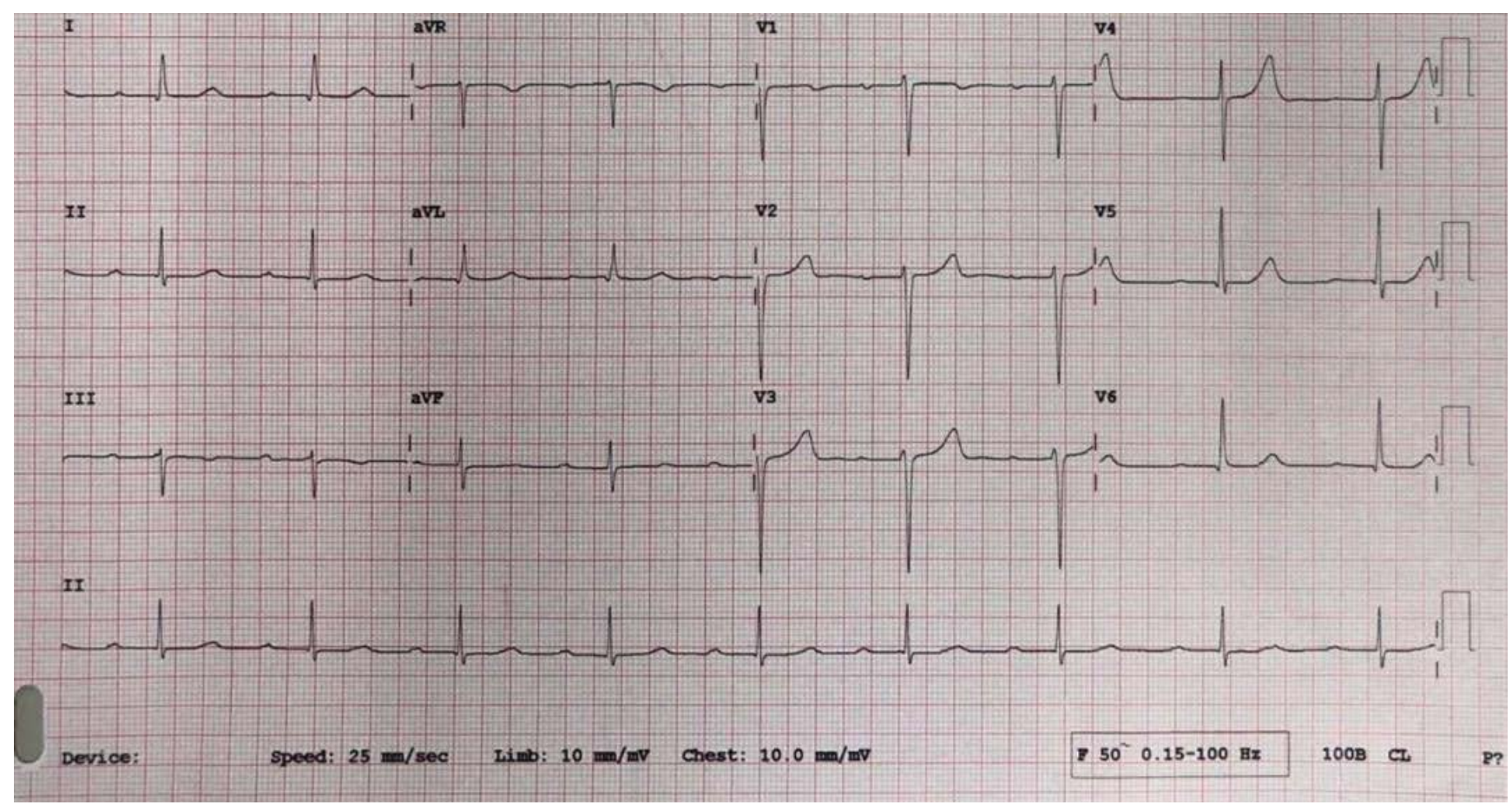

Figure 1: 12 lead ECG at presentation.

$1^{\text {st }}$ degree AV block with PR interval of $330 \mathrm{~ms}$, suggestive of AV conduction disease.

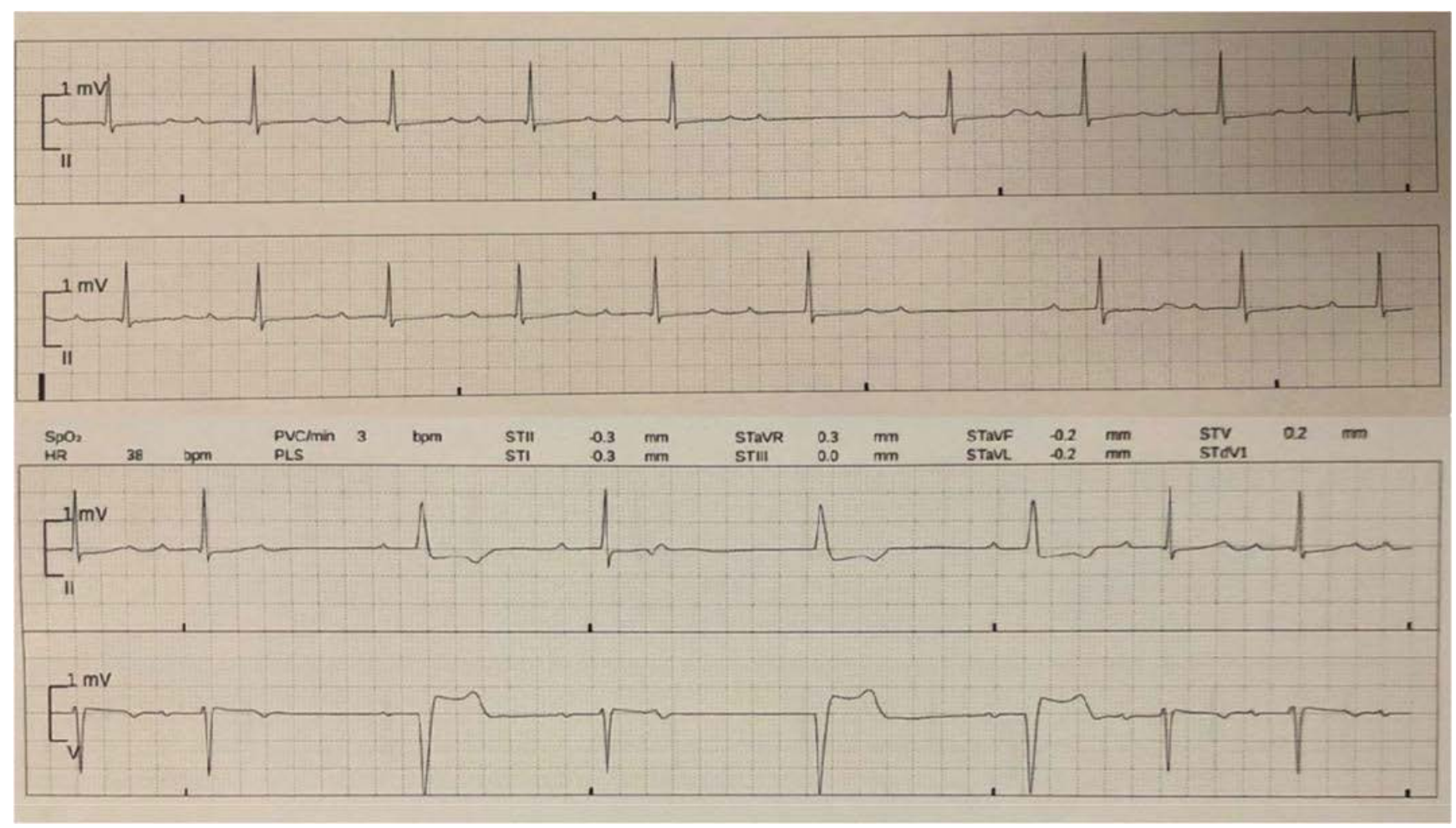

Figure 2: Telemetry interrogation with worsening bradyarrhythmias.

Increasing frequency of Wenckebach phenomenon and high-grade AV block with competing ventricular escape rhythm.

disc osteophyte complex, no definitive masses or evidence of malignancy. Three consecutive sets of blood cultures from the first three days of admission returned positive for an identical isolate of Vagococcus fluvialis, sensitive to penicillin (MIC $0.5 \mathrm{ug} / \mathrm{L}$ ) and gentamicin.

Transthoracic echocardiogram on day 0 of admission revealed normal LV size and systolic function, thickened bioprosthetic aortic valve leaflets with no obvious vegetation, no aortic regurgitation and no obvious aortic root abscess.

\section{Management}

Our patient was admitted under the cardiology service and commenced on empiric antibiotic therapy for 
presumed infective endocarditis, before being rationalised to ampicillin $2 \mathrm{~g} 4$ hourly and gentamicin $80 \mathrm{mg}$ TDS once formal culture and sensitivity results were known. Telemetry within the first 48 hours of admission demonstrated increasing sinus bradycardia with multiple episodes of transient asymptomatic Mobitz I and II AV block during wake and sleep (Figure 2). A repeat transthoracic echo was performed on day 2 of admission with significant progression of findings including reduced aortic valve leaflet motion, significant increase in echolucent space around aortic annulus with colour flow into potential paravalvular fistula, mild to moderate central aortic regurgitation, and a rocking prosthetic aortic valve. Doppler assessment of the aortic valve revealed dramatically increased measurements including peak velocity of $5.1 \mathrm{~m} / \mathrm{s}$, peak gradient of $104 \mathrm{mmHg}$ and mean gradient of $66 \mathrm{mmHg}$ (Figure 3).

Transoesophageal echocardiogram was promptly performed which confirmed the findings on transthoracic echocardiogram. A large echodense space around the heterograft was identified, consistent with abscess and fistula into the left ventricular outflow tract, in addition to a large echodensity of the bioprosthetic valve leaflets, and valve annular rocking (Figure 3 and Figure 4).

An urgent cardiothoracics consult was sought given the rapidly progressing nature of his valvular and conduction disease, and the high likelihood of treatment fai- lure with medical therapy alone. During this period, our patient continued to exhibit signs of sepsis with fevers, rigors and tachycardia consistent with septic showers, however, he did not require any invasive haemodynamic or chronotropic support at any stage. On day 8 after his emergency department presentation, a decision was made to take our patient to theatre for cardiothoracic intervention. He underwent a third redo-sternotomy, aortic valve replacement ( $27 \mathrm{~mm}$ Mosaic porcine valve), bovine pericardial patch reconstruction of the left ventricular outflow tract and insertion of a dual chamber epicardial permanent pacemaker. Intraoperative findings noted a $1 \mathrm{~cm}$ vegetation in the ascending aorta at the site of aortotomy, grossly infected aortic valve prosthesis with multiple vegetations, an abscess cavity in the aortic root anterior to the valve prosthesis in the area of the membranous septum, and an aortic annulus completely destroyed by vegetations. Gram positive cocci were appreciated on gram stain from intraoperative specimens but no organism was able to be cultured. Our patient's postoperative course was unremarkable, spending one day only in cardiothoracics intensive care before being returned to the general ward to complete six weeks intravenous ampicillin and gentamicin, in addition to beginning glecaprevir/pibrentasvir for 12 weeks with curative intent for his hepatitis $C$. Unfortunately given the rapidly progressive nature of his endocarditis, an MRI spine was unable to be performed prior to sur-

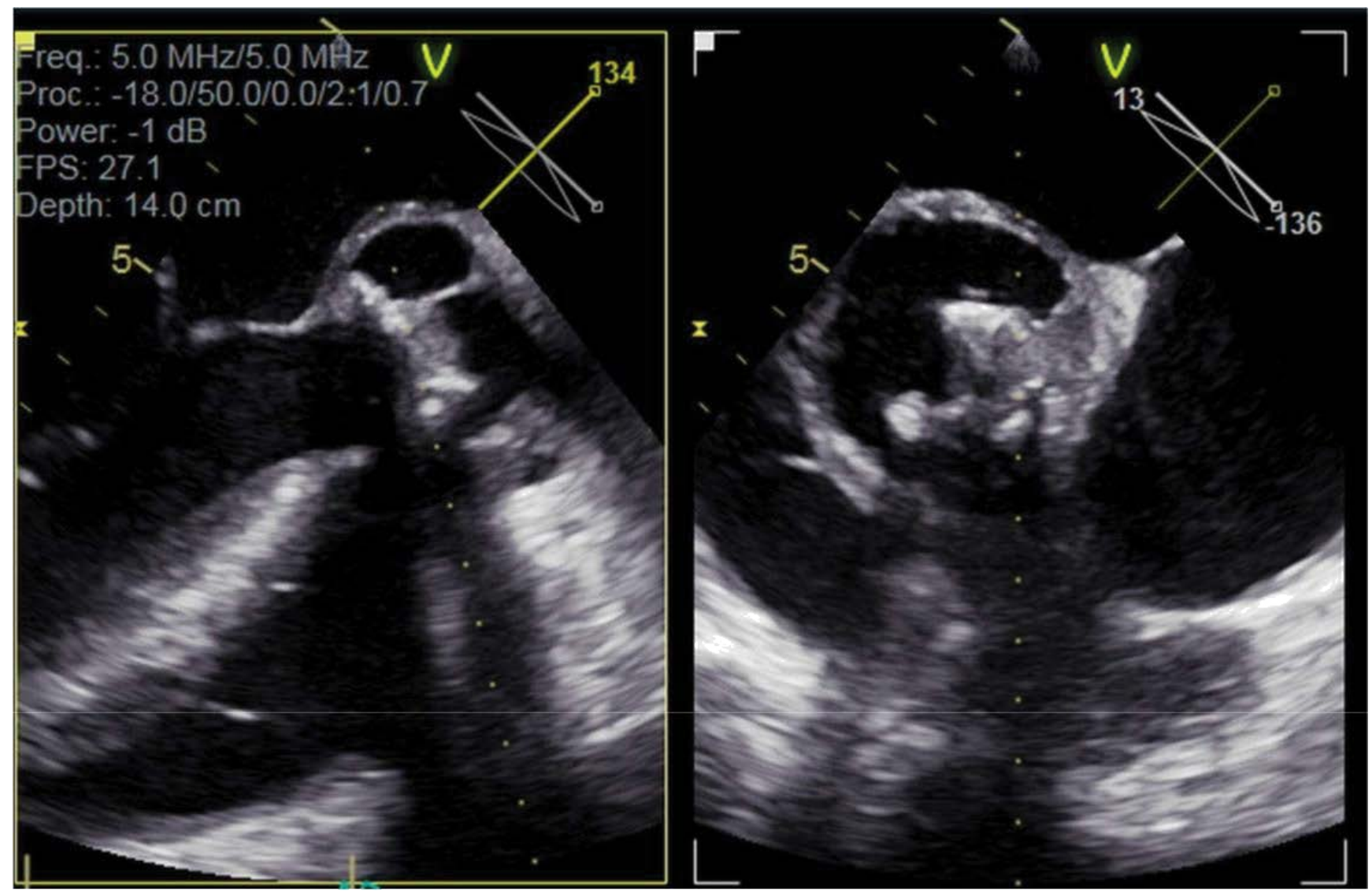

Figure 3: Transoesophageal echocardiogram multiplane images through aortic valve and aortic root.

Large echodense space around Mosaic heterograft consistent with aortic root abscess, in addition to multiple gross echo densities on bioprosthetic valve leaflets. 


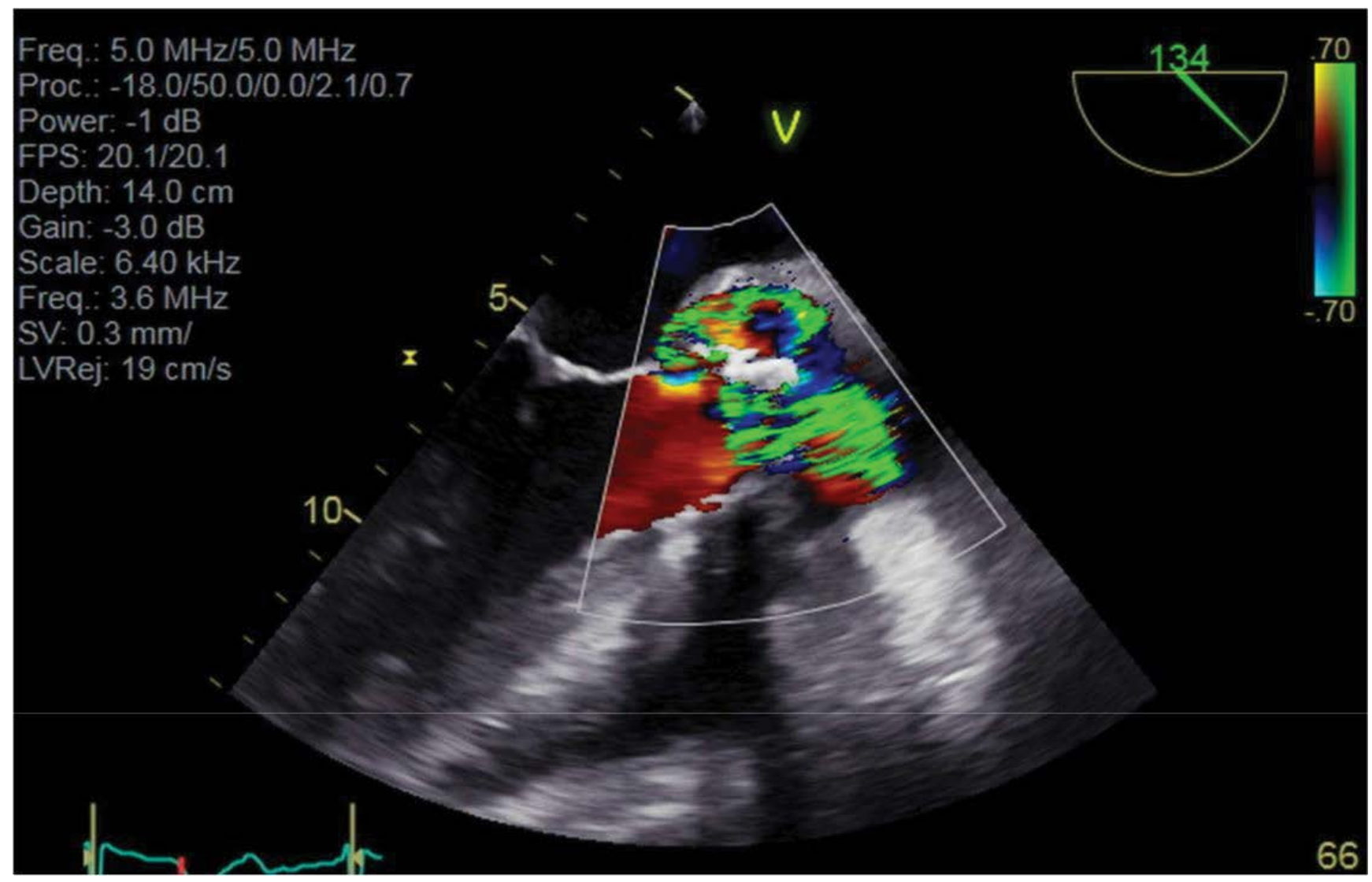

Figure 4: Turbulent doppler colour signal through infected heterograft.

High doppler velocity signals both centrally through the prosthetic aortic valve and through anterior peri-valvular space consistent with aortic root fistula into LVOT.

gery and PPM insertion to exclude discitis or osteomyelitis. A whole-body gallium scan with tomography was arranged postoperatively as a substitute investigation, however, returning negative with no abnormal gallium uptake to suggest discitis or vertebral osteomyelitis. Our patient's back pain improved rapidly with directed therapy at infective endocarditis.

\section{Discussion}

Infective endocarditis continues to be a challenging cardiac disease, with clinical courses varying from stable recovery after a prolonged antibiotic course, to severe, fulminant endocardial injury with overwhelming sepsis, embolic phenomenon, and cardiac decompensation. The contributing factors to these varied presentations are complex and include a multifaceted interplay between the causative pathogen, duration of illness prior to diagnosis, underlying structural heart disease and competing comorbidities [1].

The gram-positive cocci of staphylococcus, streptococcus and enterococcus species are known to cause approximately $80-90 \%$ of infective endocarditis, with smaller numbers secondary to the HACEK organisms and Candida [2]. Of these organisms, infective endocarditis secondary to Staphylococcus aureus is more frequently associated with complications than other pathogens, including stroke, systemic emboli, persistent bacteraemia and mortality [3]. Little is known regarding the significance of infective endocarditis secondary to the Vagococcus species.

Vagococcus fluvialis, a species of gram-positive catalase-negative cocci, is a relatively recently described organism in clinical microbiology. It was first isolated in both river water and chicken faeces in 1974 as a suspected 'unidentified enterococcus' species before being formally recognised as a distinct genus in 1989 $[4,5]$. Vagococcus fluvialis has been consistently isolated from oropharyngeal lesions of various domestic animals including pigs, cats, cattle and horses, however its role in human pathology remains unclear [6]. Al-Ahmad, et al. described the first incidence of Vagococcus fluvialis in human disease in 2008 with isolates identified from periodontal infection [7].

Infective endocarditis secondary to Vagococcus fluvialis is rarely described in the literature. To our knowledge, only two published case reports exist from 2016 and 2019, both describing a fulminant clinical course characterised by early valvular insufficiency and local tissue destruction [8,9]. The first involved a young woman with a history of intravenous drug use who presented septic with a right occipito parietal infarct and vegetations involving both aortic and mitral valves with severe insufficiency on both valves. This lady required urgent cardiothoracic intervention for both aortic and mitral 
bioprosthetic valve repair and pericardial patch for aortic defect. The second case documented a 70-year-old gentleman with a history of coronary artery bypass presenting with high grade fevers, rapidly progressive dyspnoea and multiple supra- and infravalvular vegetations involving his aortic valve with associated severe aortic insufficiency. He too was required to undergo emergency redo-do sternotomy with aortic bioprosthetic valve replacement. Both these patients completed 6 weeks antibiotic therapy following their cardiothoracics procedures and were discharged from hospital well. As with our patient, the modes of acquisition of this pathogen remain unclear.

The case we have presented is consistent with this experience, with a requirement for early surgical intervention due to prosthetic valve dehiscence resulting in fistulous connection between the LVOT and aortic root and gross valvular destruction at surgical inspection. In addition, our patient also developed progressive AV nodal conduction disease as a complication of aortic valve endocarditis. In patients undergoing early surgical intervention for infective endocarditis with the need for permanent pacing, it is important to consider that complete sterility may not be possible at the time of surgery. Therefore, a standard transvenous pacing system may not be the optimal modality. In our case, a permanent epicardial pacemaker was implanted at the time of surgery to mitigate the potential risk of infective colonisation of the pacemaker lead.

Although the documented incidence is limited, $\mathrm{Va}$ gococcus fluvialis endocarditis has been associated with rapid endocardial necrosis and valvular insufficiency in all cases described. Furthermore, our case also identifies the potential for intracardiac abscess with subsequent conduction disease. All cases have required emergent cardiothoracic intervention.

\section{Conclusion}

Vagococcus fluvialis is a rare cause of infective endocarditis. It appears to have a fulminant and rapidly progressive course with extensive endocardial necrosis and valvular insufficiency, and behaves markedly different from other lactobacilli with which it phenotypically resembles. Early recognition of Vagococcus fluvialis as a cause of endocarditis is critical due to its rapidly progressing course and thus need for early access to cardiothoracic services, valve replacement and cardiac pacing options.

\section{Learning Objectives}

Vagococcus fluvialis is a rare but recognised cause of infective endocarditis with the potential for rapidly progressive valvular damage.

Patients with suspected Vagococcus fluvialis endocarditis should be managed in early consultation with cardiothoracic surgeons in anticipation of requirement for prompt valvular intervention.

\section{Authors Declaration}

The authors have no conflicts of interest to declare.

The aforementioned authors have contributed equally to this manuscript.

\section{References}

1. Hoen B, Duval X (2013) Infective Endocarditis. New England Journal of Medicine 368: 1425-1433.

2. Selton-Suty $C$, Célard M, Le Moing V, Doco-Lecompte $T$, Chirouze C, et al. (2012) Preeminence of Staphylococcus aureus in infective endocarditis: A 1-year population-based survey. Clin Infect Dis 54: 1230-1239.

3. Fowler Jr VG, Miro JM, Hoen B, Cabell CH, Abrutyn E, et al. (2005) Staphylococcus aureus endocarditis: A consequence of medical progress. JAMA 293: 3012-3021.

4. Hashimoto H, Noborisaka R, Yanagawa R (1974) Distribution of motile Streptococci in feces of man and animals and in river and sea water. Nippon Saikingaku Zasshi 29: 387-393.

5. Collins MD, Ash C, Farrow JA, Wallbanks S, Williams AM (1989) $16 S$ ribosomal ribonucleic acid sequence analyses of lactococci and related taxa. Description of Vagococcus fluvialis gen. nov., sp. nov. J Appl Bacteriol 67: 453-460.

6. Pot B, Devriese LA, Hommez J, Miry C, Vandemeulebroecke K, et al. (1994) Characterization and identification of Vagococcus fluvialis strains isolated from domestic animals. J Appl Bacteriol 77: 362-369.

7. Al-Ahmad A, Pelz K, Schirrmeister JF, Hellwig E, Pukall R (2008) Characterization of the first oral vagococcus isolate from a root-filled tooth with periradicular lesions. Curr Microbiol 57: 235-238.

8. Abuzaanona A, Al Darzi W, Abdul Nour K (2016) Vagococcus fluvialis causing severe infective endocarditis presenting with embolic stroke: A case report. In: 11th Annual Cardiology Summit. Philadelphia, USA.

9. Jadhav KP, Pai PG (2019) A rare infective endocarditis caused by Vagococcus fluvialis. J Cardiol Cases 20: 129131. 